\title{
Evolutionary computing based hybrid bisecting clustering algorithm for multidimensional data
}

\author{
K APARNA \\ Department of Computer Applications, BMS Institute of Technology and Management, Bengaluru, India \\ e-mail: aparnak@bmsit.in
}

MS received 15 January 2017; revised 17 May 2018; accepted 17 September 2018; published online 1 February 2019

\begin{abstract}
The emerging technologies and data centric applications have been becoming an integral part of business intelligence, decision process and numerous daily activities. To enable efficient pattern classification and data analysis, clustering has emerged as a potential mechanism that classifies data elements based on respective feature homogeneity. Although K-Means clustering has exhibited appreciable performance for data clustering, it suffers to enable optimal classification with high dimensional data sets. Numerous optimization efforts including genetic algorithm (GA) based clustering also require further optimization to avoid local minima issues. In this paper, an improved Canonical GA based Bisecting K-Means algorithm (CGABC) has been developed. The proposed model incorporates min-max normalization based feature normalization of the high dimensional data sets, which is followed by T-Test analysis that significantly reduces data dimensions based on feature similarity of the data elements. The fitness value has been assigned based on inter-cluster (heterogeneous distance) and within-cluster (homogeneous distance) distances. To enable optimal features and process parameter selection, particularly cluster centers information, the conventional GA has been modified by applying multistage reproduction process, enhanced crossover and mutation. By incorporating the optimized cluster center information the Bisecting K-Means clustering has been performed, which has exhibited optimal solution for highly accurate and efficient clustering with high dimensional data sets.
\end{abstract}

Keywords. Bisecting K-Means; clustering; feature normalization; T-test analysis; modified genetic algorithm; multidimensional data sets.

\section{Introduction}

The high pace increase in technologies, data centric applications and related data gathering have demanded certain potential approaches for mining and classification tasks to enable effective data analysis. Data clustering is one of the predominant approaches for pattern (data elements) classification and grouping. Clustering represents an unsupervised pattern classification approach. Typically, the partition possesses two predominant characteristics. These are homogeneity and heterogeneity. The homogeneity within the cluster states that those data elements belonging to the same cluster must be similar. On the contrary, heterogeneity between clusters refers to those data belonging to different clusters and they must be distinct and should have different characteristics. A number of approaches require pre-defined clustering parameters such as the shape of cluster and number of clusters [1]. On the other hand, in practical applications, the number of clusters in certain dataset cannot be precisely known. There are a number of algorithms proposed for clustering, such as K-Means clustering, Fuzzy-C-Means, etc. Interestingly, majority of the existing approaches are unable to perform optimal pattern classification and natural grouping from all the input patterns. This problem becomes severe when the number of clusters used to be large in the high dimensional datasets. Generally, such limitations are because of inappropriate selection of the cluster centers. Numerous intricate issues like these are stated as unsupervised clustering, and usually are dealt with by means of certain evolutionary computing technique. Recently GA has emerged as one of the most efficient techniques for parametric optimization and sub-optimal solution retrieval [2-4]. Researches reveal that GA based clustering can be a potential technique for clustering due to its optimal parametric (centre information) selection features [5].

K-Means algorithm has been the preferred clustering approach in many applications; however the computational complexity and time consumption motivated researchers to develop its enhanced variant called Bisecting K-Means (BKM) clustering algorithm. Even being an effective alternative for data clustering, BKM suffers from computational complexity, particularly with high dimensional datasets. As stated above, the predominant reason for such limitations could be the inappropriate data center selection 
for clustering. To alleviate such limitations and enhance the BKM clustering algorithm, in this paper a novel modified evolutionary computing (EC) algorithm called Canonical Genetic Algorithm (CGA) has been proposed that performs optimal cluster parameter selection for further clustering. The considerable limitation in the existing GA algorithm is the computational time and hence requires optimization across the process to yield optimal solution towards optimized clustering. In this paper, the conventional GA has been modified at different process level so as to alleviate the existing computational time consumption issues.

Considering functional behaviour of the traditional K-Means clustering or even BKM approach where the cluster mean is selected as the center, also called centroid, it is inevitable to estimate the distance between data index or points and its cluster center. Unfortunately, in conventional GA, these distance parameters are required to be estimated each time so as to calculate fitness value and consequently it makes the overall process time consuming. To alleviate such limitations, our proposed method introduces a look-up table that stores the distances between all the data point (pairs) in advance and thus the distances are required to be estimated only once.

The general question asked associated to the cluster verification issues, is whether the essential hypothesis including shape and number of clusters, process conditions, etc. of the clustering technique are contented and opt for all of the data elements. To deal with such situations, a number of measures like the Dunn index, the XB index (Xie-Beni index), the BM index [6] and the DB index [7] have been proposed [1, 6-8]. It is a fact that it is not feasible to answer each query without having prior data information; still certain approaches can be explored to facilitate significant clustering outcomes in terms of homogeneity existing within clusters and heterogeneity between the clusters. Literature [9] reveals that in spite of exhibiting better stripshaped clustering, Dunn index degrades the overall process. Similarly, XB index exhibits poor efficiency with high dimensional data and the BM index intends to create only two clusters for majority of the data sets. On the contrary, the DB index that is defined as the ratio of the sum of the scatter within the clusters to the scatter between the clusters can be a potential measure to validate clusters, especially for high dimensional data. In this paper, the DB index and its variant (homogeneity and heterogeneity) based approaches have been applied for cluster validation. In this paper, these parameters have been used as the fitness value to perform EC (GA) based clustering. In this paper, initially, the multi-dimensional data features have been normalized using min-max normalization which has been followed by the T-Test analysis that exhibits dimensional reduction significantly to ensure optimal clustering process. By performing the T-Test analysis, the proposed modified canonical GA algorithm has been executed to perform optimal cluster parameter retrieval which has been followed by Bisecting K-Means algorithm for high dimensional data clustering.

In the remaining sections, section 2 discusses the related work, which is followed by the proposed evolutionary computing based Bisecting K-Means algorithm in section 3. Section 4 discusses the results and conclusion and future scopes are discussed in section 5 .

\section{Related work}

To enable a better alternative for clustering multi-feature large data sets, researchers in [10] developed a GA based clustering scheme. Implementing a common cost function for text as well as categorical values, they obtained the within cluster dispersion matrix to perform clustering. To enable optimal clustering, they implemented GA for cost function optimization. To enable better performance with minimum computational cost, authors in [11], have proposed a GA based clustering that incorporated clustering accuracy as the stopping criteria for GA implementation. In conventional K-Means clustering, the number of clusters is predefined or known. However, in practical cases the numbers of clusters are often unknown. To deal with this a new approach named spectral clustering was proposed in [12], but its efficiency primarily depends on the spectral relaxation that questions universal accurate solution. To alleviate such limitations, they developed a GA based algorithm to obtain optimal cluster information. They introduced a new approach by incorporating group number coding chromosome, uniform crossover operator and exponential mutation rate to perform clustering. Generally, for high dimensional data the selection of features have significant impact on clustering efficiency. Researchers in [13] developed a GA based feature selection algorithm that explores potential feature subsets including cluster centers and candidate features for clustering.

A calibrated effort was in [14], where an Antbased Template Mechanism (Ant-TM) was developed for data clustering. Researchers developed a hybrid model using Ant-TM and K-Means clustering algorithm that exhibited significantly enhanced convergence process. The prime contribution of this paper was the avoidance of local optima. Similarly, a hybrid clustering scheme using Fuzzy logic and GA was developed in [15]. In this approach the objective functions intends to enhance the conformity between the ensemble members while reducing the disagreement between clusters. To perform clustering in multi-dimensional data sets or heteroscaled dimensional data sets, a weighted GA based clustering approach was developed in [16]. A GA based K-Means algorithm was proposed in [17] that focussed on feature variables selection by reducing allied objective function. They intended to incorporate the significances of GA as well as K-Means that enabled global convergence. 
Researchers [18] proposed a canonical variate analysis for dimensional reduction in high-dimension dataset, which was then followed by clustering using a modified KMeans clustering algorithm named Hybridized K-Means Clustering Algorithm (IHKMCA). Similarly, an ant colony optimization (ACO) based clustering was proposed in [19] that employed a neighbourhood construction mechanism and data set reduction. In addition, they incorporated two objective functions named relative distance and adjusted compactness. Each of these objective functions examines the clustering solution in relation with the local neighbourhood features. This approach enabled quality estimations of numerous clustering solutions without incorporating a priori data. ACO-C comprises two preprocessing phases, neighbourhood construction and data reduction, where the first one performs removal of the local features of the data points, while the second deals with the scalability issue. The multi-objective assessment significantly enhances the retrieval of the random-shaped clusters having density differences. To deal with high dimensional data clustering, in our previous research [20] we developed a new partitional algorithm called HBKMeans (High Dimensional Bisecting K-Means) algorithm that partitions data into two parts while performing clustering so as to enable swift and accurate data clustering and process. In our research continuation, a novel stability and Mean Square Error (MSE) based Constraint based High-dimensional Bisecting K-Means clustering (CHB-KMeans) was developed in [21, 22]. With an objective to exploit the advantages of the evolutionary computing algorithm, GA and its further optimization, efficient dimensional reduction, optimal feature selection algorithms, and BKM clustering algorithm, in this paper a highly robust hybrid model has been developed that enables optimal clustering process for large scale multidimensional data sets.

\section{Our contributions}

In this paper, a highly robust and efficient evolutionary computing algorithm, named genetic algorithm based bisecting K-Means clustering scheme has been developed for multidimensional datasets. The overall proposed algorithm is presented in figure 1 .

A number of researches have revealed that Bisecting K-Means clustering can be a potential alternative for clustering and associated application such as classification and prediction. Especially, Bisecting K-Means clustering can have vital role in multi-dimensional data clustering. With these motivations, in this paper, a highly robust and efficient evolutionary computing based Bisecting K-Means clustering scheme has been developed. The overall proposed system and its implementation are discussed as follows.

\subsection{Feature normalization}

To enable efficient computation, especially for multidimensional datasets the normalization of data becomes significant. In this paper, the input features values or the data input values have been normalized over the range of $[0,1]$ using min-max normalization technique. Consider that $X_{i}$ be the $\mathrm{i}^{\text {th }}$ feature of the dataset $\mathrm{X}$, and $\mathrm{x}$ be an element of $X_{i}$. Then the normalization of the data element $\mathrm{x}$ can be performed using following equation:

$$
\operatorname{Normalized}(x)=\frac{x-\operatorname{Min}\left(X_{i}\right)}{\operatorname{Max}\left(X_{i}\right)-\operatorname{Min}\left(X_{i}\right)}
$$

where, $\operatorname{Min}\left(X_{i}\right)$ and $\operatorname{Max}\left(X_{i}\right)$ represent the minimum and maximum feature values, respectively. In case of $\operatorname{Min}\left(X_{i}\right)=\operatorname{Max}\left(X_{i}\right)$, the normalized value is assigned as 0.5 .

\subsection{T-test based feature selection}

Generally, there are two predominant issues with the multidimensional data. The first problem is the "curse of dimensionality," that states that the number of features are much higher than the number of samples, while the second problem states the fact that there can be numerous features having minimal impact on the classification result. These two significant issues somewhere influence the overall clustering and associated classification process. Since, the proposed method intends to perform Bisecting K-Means clustering for multidimensional data, to deal with aforementioned issues, in this paper a novel feature selection approach called T-test Analysis has been applied. Unlike conventional approaches and major existing systems, in this paper selecting the features using T-test intends to significantly reduce the dimension of the data by finding a small set of important features which can facilitate better classification performance. An extensively employed filter approach for data is to apply a univariate criterion distinctly on the individual features, while considering the assumption that there is no interaction between features. Here, in the implemented approach, a two-class problem test is considered. A null hypothesis $\left(H_{0}\right)$ is that the means of two populations are equal, i.e., there exist no significant differences between their means and hence revealing the fact that both the features are almost the same. It also states that these similar features do not influence the classification results significantly and hence such features can be effectively discarded. On the other hand, the features having significant difference can affect classification and hence are accepted for further processing. Therefore, in this paper the null hypothesis $H_{0}$ has been rejected and the alternate hypothesis $\left(H_{1}\right)$ has been accepted. In this paper, the T-test has been applied on individual feature, which has been followed by the comparison of the subsequent $P$ value (or the absolute values of $t$-statistics) for each feature as a 


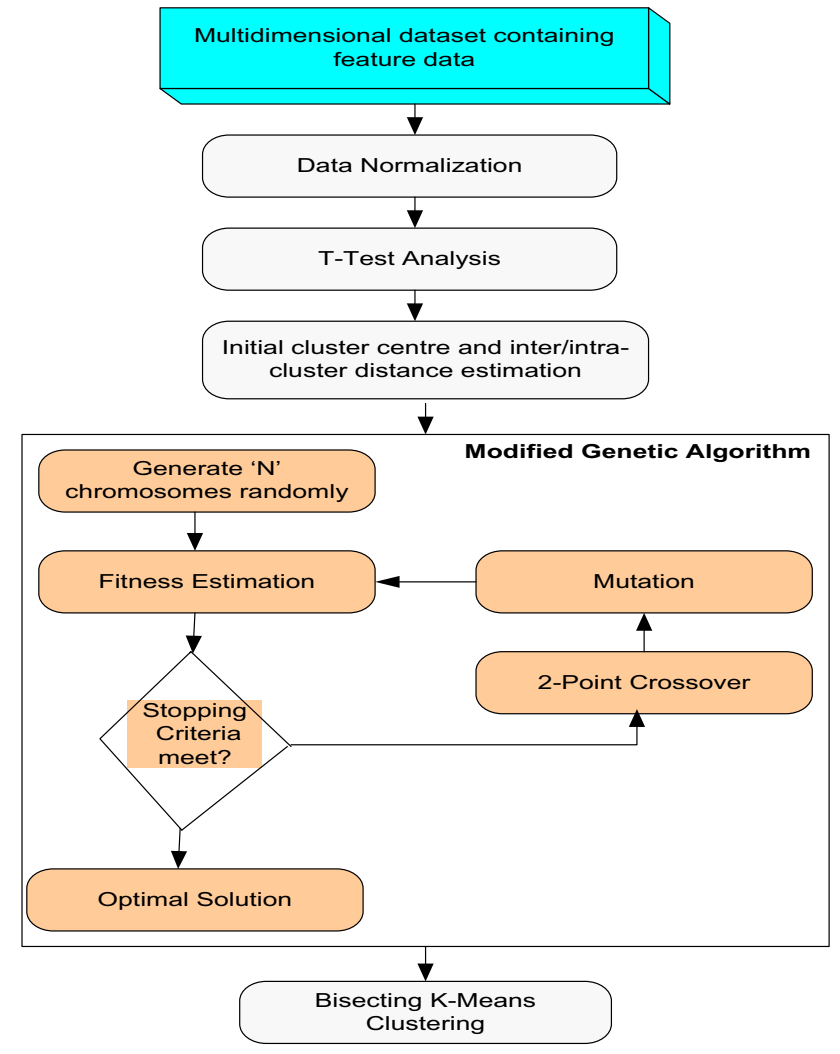

Figure 1. Proposed CGABC based bisecting K-means clustering.

measure of how effective it is at separating groups. Performing the T-test Analysis based dimensional reduction and feature selection, in this paper a robust evolutionary computing algorithm, Canonical Genetic Algorithm (CGA) based BKM algorithm called CGABC has been developed to perform clustering. The discussion of the proposed CGABC algorithm is given as follows.

\subsection{Enhanced genetic algorithm based cluster information retrieval}

The Canonical Genetic Algorithm (CGA) represents a probabilistic algorithm that maintains the population of the chromosomes, also called population individuals $P=\left\{C h_{1}, C h_{2}, \ldots, C h_{P}\right\}$, where the individual signifies certain efficient solution to a problem. Here, the individual solution $C h_{i}$ is estimated so as to facilitate certain measure of its "fitness value", which is then followed by addition of certain new population by selecting the individuals with better fitness value. This process is called as reproduction. Meanwhile, few members of the updated population undergo the consecutive process of crossover and mutation, where it forms certain new solutions. Thus, following the philosophies of natural selection and human genetic behaviour, after certain iterations, also called generations the algorithm gets converged and thus the best individual with optimal fitness and optimum solution is obtained. The implementation phases of the proposed CGABC algorithm are discussed as follows.

3.3a Binary data presentation: In this paper, the data points associated with the dataset have been considered as the participants or the candidates for the cluster centers. Here, we have considered the length of chromosome equal to the size of the dataset and the $i^{\text {th }}$ gene of a chromosome signifies the $\mathrm{i}^{\text {th }}$ data point. In our proposed clustering scheme, for certain data point with respective index $\mathrm{i}$ to be the potential candidate for a cluster center, the consequent $i^{\text {th }}$ gene is assigned the rank " 1 "; otherwise " 0 ". In our proposed model, the total number of clusters, $\mathrm{K}$ has been selected in the range of $\left[\mathrm{K}_{\min }, \mathrm{K}_{\max }\right]$, where $\mathrm{K}_{\min }$ is selected as 2 , while $K_{\max }$ is selected to be $1 / 2$ or $\sqrt{1}$. Here, 1 represents the length of chromosome.

$3.3 \mathrm{~b}$ Population initialization: Consider $\mathrm{P}$ be the population size and $\mathrm{Ch}_{\mathrm{r}}$ be the population comprising $r$ chromosomes $(\mathrm{r}=1,2, \ldots, \mathrm{P})$. Here, an integer value $\mathrm{K}_{\mathrm{r}}$ is selected randomly from $\left[\mathrm{K}_{\min }, \mathrm{K}_{\max }\right]$ and the gene associated with the index of selected data points is assigned the rank " 1 "; while the remaining genes are ranked " 0 " individually.

3.3c Fitness function evaluation: This section discusses the fitness estimation schemes for our proposed clustering approach.

\section{DB-Index based fitness Estimation}

To introduce the significant novelties in the proposed Bisecting K-Means clustering scheme is to divide a data into different clusters on the basis of two features; first the homogeneity existing within the clusters, and second the heterogeneity existing in between different clusters. To validate the clusters, numerous measures have been proposed $[1,11,12]$. Among them, DB index can be stated to be the most effective. In this paper, we have implemented DB index which facilitates efficient measure to validate the cluster formed. The dispersion measure of a cluster $C_{i}, i=1, \ldots, K_{r}$, existing in $\mathrm{Ch}_{\mathrm{r}}$ can be presented as follows (2).

$$
S_{i, q}=\left(\frac{1}{\left|C_{i}\right|} \sum_{x \in C_{i}} x-z_{i 2}^{q}\right)^{1 / q}
$$

where, $z_{i}$ represents the centre of the cluster $C_{i}$.

The highest possible intra-cluster similarity index, i.e., the similarity between the cluster $C_{i}$ with other cluster is given by a variable $\mathrm{R}_{\mathrm{i}, \mathrm{q}}$, which is mathematically given as (3):

$$
R_{i, q t}=\max _{j, j \neq i}\left\{\frac{S_{i, q}+S_{j, q}}{d_{i j, t}}\right\}
$$

In the above equation, $d_{i j, t}=d\left(C_{i}, C_{j}\right)$ represents a distance vector called Minkowski distance between the cluster 
$C_{i}$ and $C_{j}$. Here t represents the order. Finally, the DB index $\mathrm{DB}_{\mathrm{r}}$ for a chromosome $\mathrm{Ch}_{\mathrm{r}}$ is given by the following equation

$$
D B_{r}=\frac{1}{k_{r}} \sum_{i=1}^{k_{r}} R_{i, q t}
$$

Here, in the proposed evolutionary computing based Bisecting K-Means clustering scheme, the fitness of the chromosome is given by the inverse of the $\mathrm{DB}$ Index $\mathrm{DB}_{\mathrm{r}}$ of a chromosome.

$$
\text { Fitness }\left(C h_{r}\right)=\frac{1}{D B_{r}}
$$

or simplistic implementation, in this paper the value of $\mathrm{q}$ and $t$ has been assigned as 1 and 2, respectively.

\section{Inter/Intra cluster distance based Fitness Estimation}

In our proposed clustering model a novel fitness estimation model has been derived from DB Index measure in terms of homogeneous $\left(H_{O D}\right)$ and heterogeneous distances $\left(H_{T D}\right)$ between each pair of data points. Here, $H_{O D}$ represents the summation of Euclidean distance of each pair of data within the cluster and $H_{T D}$ signifies the distance between two clusters. On the basis of estimated $\left(\mathrm{H}_{\mathrm{OD}}\right)$ and $\left(\mathrm{H}_{\mathrm{TD}}\right)$ values, the fitness has been estimated as

$$
\text { Fitness }\left(\mathrm{Ch}_{r}\right)=\frac{\text { Heterogeneous Distance }\left(H_{T D}\right)}{\text { Homogeneous Distance }\left(H_{O D}\right)}
$$

In our proposed model, inter/intra cluster distances $H_{O D}$ and $H_{T D}$ are stored in a lookup table so as to speed up the proposed clustering scheme.

Observing the above retrieved mathematical model, it can be found that the estimation of the fitness value for each chromosome encompasses the calculation of the intra-distance between each data points or index and associated cluster center. In conventional approach based clustering processes, the cluster mean is applied as the center of the cluster and hence the distance between individual data point and its cluster center is required to be estimated. Thus, the iterative distance computation results into significant computational complexity addition and time consumption so as to estimate the fitness value of each chromosome. Unlike conventional approach, in our proposed CGABC based clustering scheme, the cluster centers are selected directly from the data set and to perform this look-up table has been constructed that preserves the estimated distances between all pairs of data points. Our proposed approach estimates the distance between all the pair of data points and preserves in the look-up table in advance. Such novelties have enabled our proposed approach to avoid iterative computation complexities and hence during evolution process it is needed to just look up the distance values and apply it for further processes. This approach significantly reduces the computational complexity and time consumption. The implementation of our proposed evolutionary computing (EC) scheme for data clustering is discussed as follows.

\subsection{Genetic operators}

As discussed in above sections, the prime objective of this paper is to implement a novel EC scheme called modified Canonical Genetic Algorithm (CGA) for data clustering in multidimensional data, and hence the following sections discuss the different phases of CGA including reproduction, crossover and mutation and their respective significances towards effective data clustering.

3.4a Reproduction: Generally, the genetic operator called reproduction ensures it that the current population possesses the better solution than the previous population or the gene pool. In probability, more efficient solution in the present population ensures higher probable replicates in the next generation or population. To enhance the proposed CGA approach, the traditional reproduction operator has been enhanced by adding an additional process called "Winner Substitution" which is followed by the implementation of roulette wheel selection. This multistage reproduction process enhances the probability that the individuals near the fittest value retain its presence in the next generation. The proposed crossover approach at first executes the "Winner Substitution" process that enhances the probability that a chromosome near optimal solution is reproduced. At first the proposed "Winner Substitution" process generates new chromosomes $\mathrm{Ch}_{\text {new }}$ by altering the chromosome $\mathrm{Ch}_{\mathrm{M}}$ having the highest fitness value in such a manner that the individual center candidates is substituted by the data point nearest to the corresponding mean center. In the second step of the "Winner Substitution" process, if a chromosome $C h_{j}$ is selected, that is further substituted by $C h_{n e w}$, i.e., if Fitness $\left(C h_{n e w}\right)>$ Fitness $\left(C h_{j}\right)$. Such optimization significantly increases the highest fitness value of the individual generation. In addition, it optimizes the stability of the clustering results. The time consumed in the overall computation in our proposed "Winner Substitution" process is lower than the time used during the construction of the look-up table. In the next second stage, a roulette wheel having its slots sized in proportion to the fitness value is spun $\mathrm{P}$ times and thus in each time a single chromosome is selected for the new generation (population). The overall algorithm for the proposed reproduction scheme is given in figure 2 .

3.4b Crossover: In majority of the existing GA implementation, the crossover has been performed on single gene position iteratively that might result into the formation of clusters, lower than $\mathrm{K}_{\min }$. In such cases, there can be insignificant offspring and therefore requires modifications by means of exchanging the alleles over generations. In this paper, in order to alleviate the issue of the insignificant 


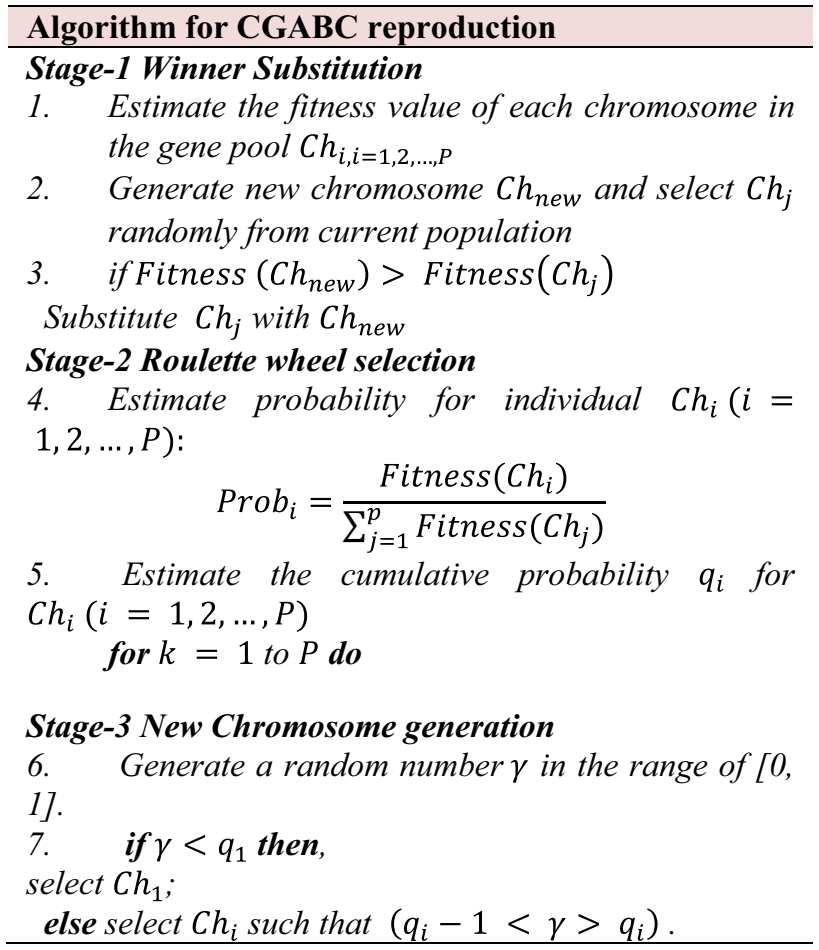

Figure 2. Algorithm for CGABC reproduction process.

clusters, an enhanced crossover operator has been applied. Let $N C_{1}$ and $N C_{2}$ be the number of clusters in the individual parent pairs, then a random integer value $\mathrm{NC}$ is created from the range $[1, \mathrm{M}]$, where $M=\operatorname{Min}\left(N C_{1}, N C_{2}\right)$. It is then followed by the random selection of the $\mathrm{NC}$ genes having allele 1's in each of the parents for crossover process. In crossover process, the parent pair exchanges their alleles at the selected gene so as to form a pair of offspring that later becomes a member of population for further computation. In this paper, 2-point crossover has been performed. The overall process of crossover in our proposed CGABC is given as follows (figure 3).

3.4c Mutation: In our proposed model, the predominant objective of mutation algorithm is to incorporate new genetic characteristics or materials in certain existing chromosome. In general, mutation takes place at certain predefined probability factor called probability of mutation $\mathrm{p}_{\mathrm{m}}$. In this paper, the smaller value of $\mathrm{p}_{\mathrm{m}}[0,1]$ has been applied so as to ensure that the good solutions could not be distorted significantly. Usually, traditional mutation process is applied on a gene-by-gene basis and thus with certain defined value of $\mathrm{p}_{\mathrm{m}}$, the individual gene in the gene pool or the population (chromosomes) undergoes mutation. In order to avoid significant distortion in the available solutions, in this paper the mutation operator has been modified by permitting only the genes satisfying the following conditions

$$
\begin{gathered}
\left(\mathrm{K}<\mathrm{K}_{\max }\right) \text { when allele }={ }^{\prime} 0^{\prime} \text {, or } \\
\left(\mathrm{K}>\mathrm{K}_{\min }\right) \text { when allele }=^{\prime} 1^{\prime}
\end{gathered}
$$

where, $\mathrm{K}$ represents the number of clusters (or 1's) for the chromosomes being assessed for mutation. Once the gene is selected for mutation process, then associated allele is modified and the allele ' 0 ' is changed to ' 1 ' or vice versa.

The overall modified algorithm for mutation is given as follows (figure 4):

The overall algorithm for the proposed approach of CGABC is summarized as follows (figure 5):

Retrieving the CGABC based clustering parameter retrieval, the proposed clustering model executes Bisecting K-Means clustering algorithm that eventually performs data clustering. A brief of our proposed Bisecting K-Means algorithm is given as follows.

\subsection{CGABC based bisecting K-means}

K-Means algorithm has been one of the most popular iterative centroid-based divisive algorithms having significant recognition for data clustering utilities. Literature study reveals that especially for high dimensional data clustering the estimation of centroid information can be of great significance for K-Means clustering. On the other hand, Bisecting K-Means has also emerged as a potential paradigm for efficient clustering utilities. With these motivations, in this paper the proposed CGABC algorithm has been incorporated with the Bisecting K-Means algorithm for high dimensional data clustering. The overall proposed Bisecting K-Means algorithm functions as follows (figure 6).

The performance of the proposed algorithm has been evaluated with different high dimensional datasets. The results retrieved are discussed in the next section of the presented manuscript.

\section{Result analysis}

In this paper, a robust Canonical Genetic Algorithm based Bisecting K-Means clustering (CGABC) has been developed for multidimensional data clustering. The overall algorithm has been developed using MATLAB2015b software tool. To examine the robustness and performance of the proposed evolutionary computing algorithm based Bisecting K-Means (BKM) algorithm CGABC, in this paper the performance assessment has been made with different multidimensional data sets. Here, data sets such as Spambase [23], Pen-Based Recognition of Handwritten Digits [24] and IRIS [25] were used for analysis. The overall performance of the proposed model has been obtained in terms of clustering accuracy, precision, recall 


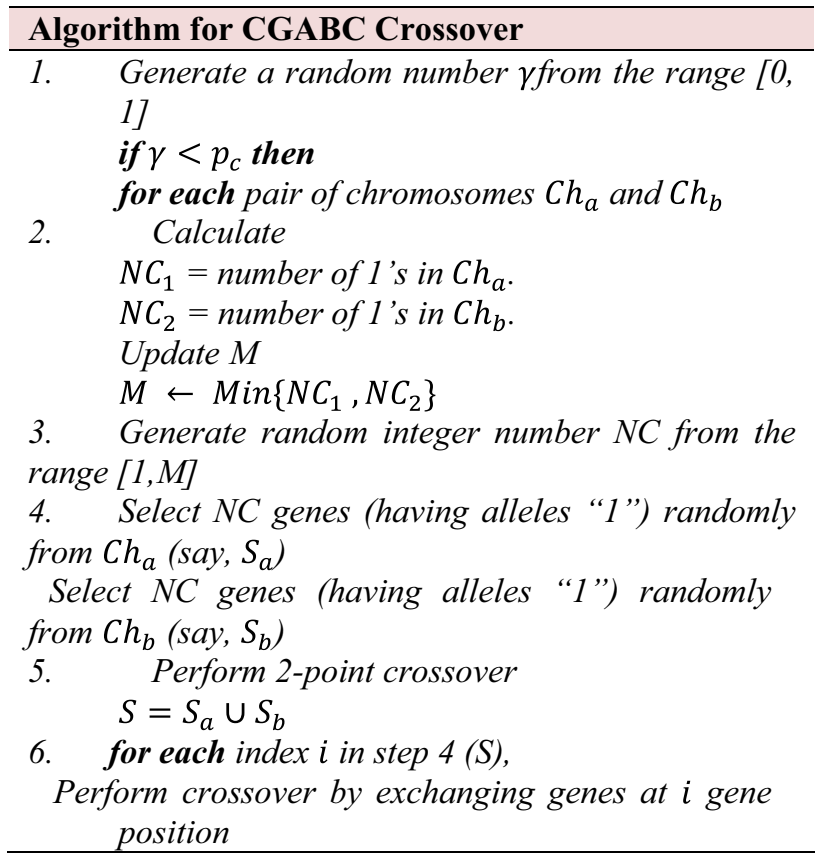

Figure 3. Algorithm for CGABC crossover process.

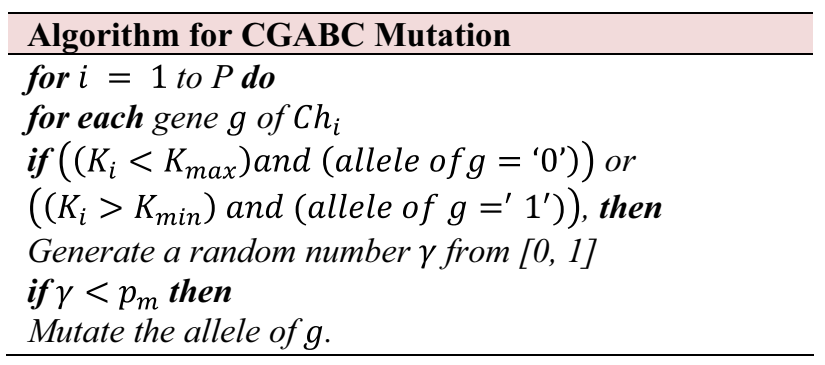

Figure 4. Algorithm for CGABC mutation process.

and F-Measure. The overall classification accuracy obtained by CGABC algorithm is given in table 1 .

The CGABC algorithm has shown improvement over other baseline experiments conducted such as HBK-Means [20], CHBK-Means [22], FGPSO [26]. This is illustrated in table 2.

In order to examine the effectiveness of the proposed CGABC Bisectional K-Means clustering algorithm, the results have been compared with other techniques. Table 3 presents a comparison chart for clustering efficacy (in terms of clustering accuracy) of the proposed algorithm and other existing approaches.

As depicted in table 3, numerous existing researches have applied UCI data sets. However, considering high dimensional data with multiple features have not been explored by major existing algorithms. Hence, to validate our proposed partitional clustering model, we have applied benchmark data sets, namely, Spambase [23], Pen [24] and
CGABC algorithm based clustering

Step-1 Perform multidimensional data selection

Step-2 Perform feature normalization of the selected data

Step-3 Perform T-Test based dimensional reduction

Step-4 Perform cluster center estimation for each chromosome

Step-5 Implement evolutionary computing CGABC algorithm for clustering

1. Generate the population randomly with size $\mathrm{N}=30$, where each chromosome contains the information about the cluster centers.

2. Estimate the Euclidean distance of each pair of data within cluster and between the clusters.

3. Estimate the fitness value of individual chromosomes using equation (5) and (6).

4. Perform ranking of the chromosomes in the gene pool based on corresponding fitness value.

5. Perform mutation by stripping the chromosomes with the minimum fitness value by the chromosomes with the maximum fitness value.

6. Perform 2-point crossover and mutation approach for offspring re-selection (Roulette wheel selection).

7. Terminate the algorithm when $95 \%$ of the chromosomes in the gene pool obtain unique fitness value, beyond this level the fitness value of chromosome get almost saturated.

Step-6 Obtain the centroid information for further clustering.

Step-7 Perform Bisecting K-Means clustering by using retrieved optimal cluster center information.

Figure 5. CGABC based clustering approach.

\section{CGABC based Bisecting K-Means Clustering}

Step-1 Select the centroid of data $\mathrm{M}$ using proposed CGABC algorithm

Step-2 Select $C_{l}$ point as initial centroid

Step-3 Calculate another centroid using CGABC

algorithm

Step-4 Divide the data M into two sub-clusters as per following conditions:

$$
\begin{aligned}
& x_{i} \in M_{A}, \text { if }\left\|x_{i}-C_{a}\right\| \leq\left\|x_{i}-C_{b}\right\| \\
& x_{i} \in M_{B}, \text { if }\left\|x_{i}-C_{a}\right\|>\left\|x_{i}-C_{b}\right\|
\end{aligned}
$$

Step-5 Re-estimate the centroid by mean value of the subclusters and iterate the process (step 1- step 4), till the desired number of clusters are not found.

Figure 6. CGABC based Bisecting K-Means Clustering.

Iris [25]. Results revealed that the proposed CGABC algorithm outperforms major existing approaches in terms of clustering accuracy. In addition, the precision of $91 \%$ affirms better feature selection and classification. The overall results obtained depict that the proposed system functions better than other approaches. 
Table 1. Efficacy analysis of CGABC clustering scheme.

\begin{tabular}{lcccc}
\hline Dataset & $\begin{array}{c}\text { Accuracy } \\
(\%)\end{array}$ & $\begin{array}{c}\text { Precision } \\
(\%)\end{array}$ & $\begin{array}{c}\text { Recall } \\
(\%)\end{array}$ & $\begin{array}{c}\text { F-Measure } \\
(\%)\end{array}$ \\
\hline Spam & 91.70 & 91.00 & 60.40 & 92.00 \\
$\quad$ base & & & & \\
Pen & 87.20 & 89.30 & 77.10 & 82.60 \\
IRIS & 79.06 & 80.01 & 89.97 & 84.80 \\
\hline
\end{tabular}

Table 2. Performance comparison of the enhanced novel algorithm with HBK-means, CHBK-Means and FGPSO with CGABC algorithm.

\begin{tabular}{lccccc}
\hline $\begin{array}{l}\text { Performance } \\
\text { metrics }\end{array}$ & Datasets & $\begin{array}{c}\text { HBK- } \\
\text { means }\end{array}$ & $\begin{array}{c}\text { CHBK- } \\
\text { means }\end{array}$ & FGPSO & CGABC \\
\hline $\begin{array}{l}\text { Clustering } \\
\text { Accuracy }\end{array}$ & $\begin{array}{c}\text { Spam } \\
\text { Base } \\
\text { Pen } \\
\text { Based }\end{array}$ & $68 \%$ & $75 \%$ & $90 \%$ & $\mathbf{9 2 \%}$ \\
& & & $78 \%$ & $78 \%$ & $\mathbf{8 7 \%}$ \\
\hline
\end{tabular}

Table 3. Performance comparison of the proposed CGABC algorithm.

\begin{tabular}{lcc}
\hline Algorithms & Data sets & Clustering accuracy (\%) \\
\hline$[21]$ & Spambase & 75.0 \\
{$[27]$} & IRIS & 90.3 \\
{$[28]$} & IRIS & 88.5 \\
{$[29]$} & IRIS & 88.6 \\
& IRIS & 63.1 \\
{$[30]$} & IRIS & 52.6 \\
Proposed CGABC & Spambase & $\mathbf{9 1 . 7}$ \\
& Pen & 87.2 \\
& IRIS & 79.06 \\
\hline
\end{tabular}

\section{Conclusion}

In this paper, a modified Canonical Genetic Algorithm (CGA) based Bisecting K-Means clustering algorithm (CGABC) has been developed. The use of min-max normalization has enabled better feature normalization that significantly has enhanced overall clustering process. The implementation of T-Test Analysis approach has significantly reduced the dimension so as to enable optimal feature selection and swift clustering process. The applied T-Test analysis algorithm has employed inter-feature similarity matrix to perform dimensional reduction and feature selection. Further, an enhanced GA algorithm has been developed for clustering parameter selection and optimization. In addition to the DB Index based cluster validation scheme, two distance parameters, homogeneous distance (distance between the each pair of data points within a cluster) and heterogeneous distance (inter cluster distance) has been introduced to estimate the fitness value of each candidate of the cluster center, while intending to reduce the heterogeneous distance and increase homogeneous distance. The use of multi-stage reproduction, 2-point crossover and mutation has enabled optimal candidate solution selection for cluster centers which has been used for further Bisecting K-Means clustering process. The performance of CGABC clustering algorithm with different multi-dimensional data sets has revealed that the proposed approach outperforms K-Means algorithm, Constraints based Bisecting K-Means algorithm (CHB-K-Means) and other GA based clustering approaches. In future, the proposed algorithm can be enhanced by exploring BKM with other evolutionary computing schemes such as ACO or PSO. Also, the selection of better fitness values and stopping criteria can also be examined to enhance clustering performance.

\section{References}

[1] Bezdek J C 1998 Some new indexes of cluster validity. IEEE Trans. Syst. Man Cybern. Part B Cybern. 28: 301-315

[2] Goldberg D E 1989 Genetic Algorithms in Search, Optimization and Machine Learning. New York, NY: AddisonWesley

[3] Michalewicz Z 1992 Genetic Algorithms + Data Structures = Evolution Programs. New York, NY: Springer-Verlag

[4] Mitchell M 1996 An Introduction to Genetic Algorithms. London: The MIT press

[5] Bandyopadhyay S and Maulik U 2002 Genetic clustering for automatic evolution of clusters and application to image classification. Pattern Recogn. 35: 1197-1208

[6] Bandyopadhyay S and Maulik U 2001 Nonparametric genetic clustering: comparison of validity indices. IEEE Trans. Syst. Man Cybern. Part C Appl. Rev. 31: 120-125

[7] Davis D L and Bouldin D W 1979 A cluster separation measure. IEEE Trans. Pattern Anal. Mach. Intell. 1: 224-227

[8] Jones D and Beltramo M A 1991 Solving partitioning problems with genetic algorithms. In: Proceedings of the 4th International Conference on Genetic Algorithms, pp. 442-449

[9] Hwei-J L, Fu-Wen Y and Yang-Ta K 2005 An efficient GAbased clustering technique. Tamkang J. Sci. Eng. 8(2): $113-122$

[10] Li J, Gao X and Jiao L-c 2003 A GA-based clustering algorithm for large data sets with mixed and categorical values. In: ICCIMA Proceedings. Fifth International Conference Computational Intelligence and Multimedia Applications, pp. 102-107

[11] Cheng C H and Wei L Y 2007 An evolutionary computation based on GA optimal clustering. In: International Conference on Machine Learning and Cybernetics 2007, Hong Kong, pp. 1821-1825 
[12] Xiaohong L and Min L 2009 GAKC: a new GA-based k clustering algorithm. In: Second International Symposium on Information Science and Engineering Shanghai, pp. 334-338

[13] Sun M, Xiong L, Sun H and Jiang D 2009 A GA-based feature selection for high-dimensional data clustering, genetic and evolutionary computing. In: WGEC '09. $3 \mathrm{rd}$ International Conference Guilin, pp. 769-772

[14] Zhang W, Chang C K, Yang H I and Jiang H Y 2010 “A hybrid approach to data clustering analysis with K-means and enhanced ant-based template mechanism," Web Intelligence and Intelligent Agent Technology (WI-IAT). In: IEEE/ WIC/ACM International Conference 2010, Toronto, pp. 390-397

[15] Razizadeh N, Badamchizaeh M A and Ghasempour M S G 2013 A new GA based method for improving hybrid clustering. In: 21st Iranian Conference on Electrical Engineering (ICEE) Mashhad, pp. 1-6

[16] Nopiah Z M, Khairir M I and Baharin M N 2009 A weighted genetic algorithm based method for clustering of Heteroscaled datasets. In: International Conference on Signal Processing Systems, Singapore, pp. 971-975

[17] Zhiwen Y and Hau-San W 2006 Genetic-based K-means algorithm for selection of feature variables. In: 18th International Conference on Pattern Recognition (ICPR'06) Hong Kong, pp. 744-747

[18] Behera H S, Rosly B and Diptendra K 2011 An improved hybridized K-means clustering algorithm (IHKMCA) for high dimensional dataset \& its performance analysis. Int. J. Comput. Sci. Eng. (IJCSE) 3(3): 1183-1190

[19] Tulin I, Sinan K and Nur E O 2015 Ant colony optimization based clustering methodology. Appl. Soft Comput. 28: 301-311

[20] Aparna K and Mydhili K N 2015 HB-K Means: An algorithm for high dimensional data clustering using bisecting
K-means. Int. J. Appl. Eng. Res. (IJAER) 10(4): 34945-34951

[21] Aparna K and Nair M K 2015 Effect of outlier detection on clustering accuracy and computation time of CHB K-means algorithm. Int. Conf. Comput. Intelligence Data Mining 2: $25-35$

[22] Aparna K and Mydhili K N 2016 Incorporating stability and error-based constraints for a novel partitional clustering algorithm. Int. J. Technol. (IJTech) 7(4): 691-700

[23] Spam base Data Set from http://archive.ics.uci.edu/ml/data sets/Spambase. Accessed date September 2016

[24] Pen-Based Recognition of Handwritten Digits Data Set from http://archive.ics.uci.edu/ml/datasets/PenBase dRecognitionofHandwrittenDigits. Accessed date September 2016

[25] Iris Dataset from http://archive.ics.uci.edu/ml/datasets/Iris. Accessed date September 2016

[26] Aparna K and Nair M K 2016 Development of fractional genetic PSO algorithm for multi objective data clustering. Int. J. Appl. Evol. Comput. (IJAEC)—IGI Glob. Publ. 7(3): ISSN: 1942-3594, EISSN: 1942-3608. https://doi.org/10. 4018/ijaec.2016070101, Indexed in ACM Digital Library and DBLP, pp 1-16

[27] Bahman A, Sattar H and Mohammad H Y 2013 Detection of outliers and reduction of their undesirable effects for improving the accuracy of K-means clustering algorithm. Int J. Comput. Appl. Technol. Res. 2(5): 552-556

[28] Chunfei Z and Zhiyi F 2013 An improved K-means clustering algorithm. J. Inf. Comput. Sci. 10(1): 193-199

[29] Madhu Y, Srinivasa R P and Srinivasa T M 2010 Enhancing K-means Clustering algorithm with improved initial center. Int. J. Comput. Sci. Inf. Technol. 1(2): 121-125

[30] Abdul N and Sebastian M P 2009 Improving the accuracy and efficiency of the k-means clustering algorithm. Proceedings of the World Congress on Engineering (I) 